http://jmscr.igmpublication.org/home/ ISSN (e)-2347-176x ISSN (p) 2455-0450

crossref DOI: https://dx.doi.org/10.18535/jmscr/v8i8.24

Journal Of Medical Science And Clinical Research

\title{
A Novel Approach to Managing Haemorrhage in Dengue Haemorrhagic Fever-A Life Saved!
}

\author{
Authors \\ Dr Pai Ashutosh A., Dr Sutay Nita R., Dr Kapari Karan \\ Department of Paediatrics, Grant Government Medical College and JJ Hospital \\ Corresponding Author \\ Dr Pai Ashutosh A.
}

\begin{abstract}
Dengue fever is one of the most commonly encountered mosquito-borne viral infections in India. It can present with a diverse clinical spectrum ranging from a simple undifferentiated fever to Dengue Haemorrhagic Fever with Multiorgan failure. Bleeding is responsible for a significant proportion of fatal outcomes in Dengue fever. We report this 10 year old female child with history of fever, bloody vomitus and passage of black coloured stools. On examination, child was Febrile with signs of Hypotensive shock, petechiae, hepatomegaly, jaundice, ascites and pedaloedema. Blood investigations revealed Dengue NS1 positive with thrombocytopenia, Hepatitis and abnormal coagulopathy. She was treated for shock with crystalloids and inotropes. Despite repeated transfusions of Fresh Frozen Plasma, Platelets and Packed Cells as recommended in guidelines, child continued to show bleeding. The Child was eventually put on Ventilator for worsening respiratory distress. Child was given a trial of Injection Octreotide infusion which was successful in stopping the bleeding, never before tried. Eventually child recovered and was discharged.
\end{abstract}

Keywords: octreotide; dengue; novel; bleeding.

\section{Introduction}

Dengue fever is a self-limiting mosquito borne arbovirus disease that is endemic to India. All 4 virus serotypes have been reported. The most striking manifestation of dengue infection is the plasma leakage syndrome, which, when severe, leads to shock. In extreme cases, multi-organ dysfunction occurs, leading to death. Although severe bleeding is generally considered an unusual manifestation of dengue, bleeding is responsible for a significant proportion of fatal outcomes. ${ }^{(1)}$ The exact pathophysiological mechanism behind bleeding in patients with dengue infection has not been clearly elucidated. Dengue patient can have bleeding with normal platelets and coagulation parameters. On the other hand, most of these haemostatic abnormalities may even spontaneously resolve without any clinically significant bleeding. ${ }^{(10)}$ As per current guidelines, Dengue Haemorrhagic Fever (DHF) can be divided into 4 grades of severity. The recommended treatment for severe bleeding in DHF is Packed Cell, Platelet and Fresh Frozen Plasma transfusion. Guidelines do not recommend any definitive measure for controlling bleeding in Dengue fever. This case is being reported to highlight the need for investigating new 
therapeutic options for controlling resistant bleeding in DHF.

\section{Case Report}

10 year old female child, 3rd By Birth Order, Resident of Jalgaon, Maharashtra, brought by parents with chief complaints of fever since 5 days, bloody vomitus and passage of black coloured stools since 1 day. The patient was previously apparently well. She presented on the 5th day of an acute febrile illness with multiple episodes of bloody vomiting and passage of black tarry stools since 1 day. On examination the child was lethargic but conscious and oriented with good central and peripheral pulses. She was neither pale nor icteric but had pedal oedemaup to the ankles. Peripheries were warm with capillary refill time of less than 3 seconds. Her pulse rate was $130 / \mathrm{min}$ and respiratory rate $40 / \mathrm{min}$. Blood pressure was between 5 th and 50th centile for age. Reduced breath sounds were noted in the right lung base. She had marked tenderness over the right hypochondrium with a hepatomegaly of $4 \mathrm{~cm}$ below the costal margin with ascites. Rest of the systemic examination was normal. 2 large bore IV Lines were secured and child was started on IV Maintenance fluid and NS @ 10cc/kg/hr. Ionotropic support was added. Chest X-ray showed moderate right sided pleural effusion with no tracheal shift. Complete blood count showed Haemoglobin $(\mathrm{Hb})$ of $8 \mathrm{~g} / \mathrm{dl}$, White cell count of 20,000/cu mm, Platelet count of $1.5 \mathrm{Lakhs} / \mathrm{cu} \mathrm{mm}$. Child was started on Inj cefotaxime and Inj Amikacin for antibacterial prophylaxis. Dengue NS1 antigen was positive. Liver Function tests showed moderately elevated SGOT/PT. IV maintenance was changed to Hepatoprotective Drip( $80 \%$ D10, 20\%NS, 1\%KCL, $1 \%$ Calcium Gluconate, $0.4 \%$ Multivitamin). Serial platelet monitoring showed declining trend with platelet count falling to as low as $1000 / \mathrm{cu} \mathrm{mm}$. PT-INR was deranged to three times the normal limit. Patient continued to have hematemesis. She even developed multiple petechiae on flexor aspect of both arms and legs. Child was transfused with multiple units of Fresh Frozen Plasma (FFP) and Platelets. With fall in $\mathrm{HCT}$ and $\mathrm{Hb}$ secondary to bleeding, Patient was transfused with total 3 units of Packed Cells (PCV). Despite all above measures, patient continued to bleed. Child was given trial of Tranexamic acid, an ant fibrinolytic agent, to partial effect. Meanwhile, due to increased respiratory distress, child had to be mechanically ventilated. Patient continued to have bleeding through nasogastric tube. Child was started on Inj Octreotide infusion at $1 \mathrm{ug} / \mathrm{kg} / \mathrm{min}$, as a novel therapy to control blood loss. Child responded and gradually hematemesis and other bleeding manifestations stopped. Platelet counts improved..Respiratory distress settled and hence child was successfully extubated. Child was treated for Ventilator associated Pneumonia with antibiotics. General condition of child bettered and eventually could be discharged.

\section{Discussion}

Dengue is the commonest arthropod-borne viral infection in humans. There are four closely related but antigenically different serotypes of the virus that can cause dengue (DEN-1, DEN-2, DEN-3, DEN-4). Dengue has a wide spectrum of outcomes from asymptomatic infection to symptomatic illness that can vary from undifferentiated fever to Dengue Haemorrhagic Fever with Multiorgan dysfunction. Although the term 'dengue haemorrhagic fever' is commonly used to denote severe disease, plasma leakage and not haemorrhage, is the hallmark of severe dengue. $^{(2)}$

Bleeding manifestationsin dengue range from minor cutaneous and mucosal bleeding to deep visceral bleeding, including intracranial, pulmonary, gastrointestinal and intraperitoneal haemorrhage. Bleeding manifestations in dengue are seen in $20-60 \%$ of hospitalized patients ${ }^{(3)}$. Bleeding is responsible for significant morbidity and mortality in Dengue infection. The mechanism by which patients with dengue infection may be more prone to bleeding is complex but the main contributors are 
coagulopathy, platelet dysfunction, thrombocytopenia, and vasculopathy ${ }^{(4)}$. On the other hand,

Major bleeding manifestations in dengue do not necessarily correlate with coagulation abnormalities. In particular, the degree of thrombocytopenia does not closely relate to the risk and severity of bleeding in dengue. ${ }^{(5)}$

Since thrombocytopeniais the most striking abnormality seen in dengue patients, platelet transfusion has been widely used, both prophylactically as well as therapeutically. The pathophysiological relationship between thrombocytopenia and bleeding in dengue is not clearly understood.

Thrombocytopenia in dengue is due to several causes. These include Bone marrow suppression together with peripheral destruction of platelets through activation of the complement cascade, antibody-mediated lysis and phagocytosis ${ }^{(6)}$.

Currently the guidelines recommend administration of fresh packed cells or fresh whole blood in patients who have moderate to severe bleeding in dengue. Although there is little evidence, Platelet transfusion is recommended in addition to the above for severe systemic bleeding. ${ }^{(7)}$ It is recommended to transfuse Fresh Frozen Plasma (FFP) in patients with coagulopathy and severe bleeding. Certain studies have evaluated role of FFP in improving platelet count but no significant outcome to prevent or stop bleeding was seen. ${ }^{(8)}$

Despite trying all of the above measures, bleeding did not stop or reduce in our patient. At present, the guidelines for the management of dengue do not recommend the use of any therapeutic agent for prevention or treatment of bleeding. Various such therapeutic agents have been studied, including recombinant activated factor VII (rFVIIa), anti-D globulin and intravenous immunoglobulin (IVIg), among others. Evidence for their use is currently insufficient ${ }^{(9)}$ The above therapeutic measures were not available in our hospital for use.
Tranexamic acid is an anti-fibrinolytic agent that preserves and stabilizes fibrin's matrix structure and thereby helps to control bleeding. Its use is currently recommended to mitigate menstrual bleeding and gastrointestinal bleeding. There are no studies on its use in dengue. Inj Tranexamic acid was given to our patient in dose of 15 $\mathrm{mg} / \mathrm{kg} /$ day TDS. No fresh new petechial lesions were seen. Hematemesis continued unabated.

Octreotide is a synthetic analogue of somatostatin, with a similar spectrum of action but with a much longer biological half-life. The various actions of somatostatin are mediated through 5 types of specific membrane receptors. The gastrointestinal effects of octreotide are inhibitory to the azygous blood flow, gastro-pancreatic secretions, and splanchnic hemodynamic and intestinal motility. Octreotide decreases the inflow of blood to portal system by constricting the splanchnic arterioles and significantly reduces intravariceal pressure and thus decreasing variceal bleed. Hence it was able to control bleeding significantly in Dengue working similar to biological off switch. Its further use should be studied in severe treatment resistant bleeding in Dengue, in particular to control hematemesis.

\section{Conclusion}

This case highlights the need to thoroughly study novel therapeutic options to prevent or control sever bleeding in dengue patients as it is the most significant indicator of mortality in such patients.

\section{References}

1. Senaka Rajapakse at al; Prophylactic and therapeutic interventions for bleeding in dengue:a systematic review; Trans R Soc Trop Med Hyg 2017; 111: 433-439

2. Dalugama, C., Gawarammana, I.B. Dengue hemorrhagic fever complicated with acute liver failure: a case report. J Med Case Reports 11, 341 (2017). https://doi.org/10.1186/s13256-017-1510

3. Laul A, Laul P, Merugumala V, et al. Clinical profiles of dengue infection 
during an outbreak in northern India. J Trop Med 2016;2016:5917934.

4. Woon YL, Hor CP, Hussin N, et al. A two-year review on epidemiologyand clinical characteristics of dengue deaths in Malaysia, 2013-2014. PLoS Negl Trop Dis 2v016;10(5): $\mathrm{e} 0004575$

5. Lum LC, Goh AY, Chan PW, et al. Risk factors for hemorrhagein severe dengue infections. J Pediatr 2002;140(5):62931.Am J Trop Med Hyg. 2001 Dec; 65(6):848-51.

6. Thomas L, Kaidomar S, Kerob-Bauchet B, et al. Prospective observational study of low thresholds for platelet transfusion in adult dengue patients. Transfusion 2009;49(7):1400-11.

7. Honda S, Saito M, Dimaano EM, et al. Increased phagocytosis of platelets from patients with secondary dengue virus infection by human macrophages. Am J Trop Med Hyg 2009;80(5):841-5.

8. Guidelines: National Vector Borne Disease Control, Ministry of Health and Family Welfare, Government of India, 2015.

9. Sellahewa KH, Samaraweera N, Thusita $\mathrm{KP}$, et al. Is fresh frozen plasma effective for thrombocytopenia in adults with dengue fever? Aprospective randomised double blind controlled study. Ceylon Med J2008;53(2):36-40

10. Gunawardane NWA, Dissanayake U, Sellahewa $\mathrm{K}$, et al. Guidelines on management of dengue fever and dengue hemorrhagic fever in adults. Colombo, Sri Lanka: Ministry of Health, 2010. 